\title{
Effect of nanoscale fillers on the viscoelasticity of polymer nanocomposites
}

\author{
Mohammad Bonakdar \\ Center for Intelligent Materials Systems and Structures, Department of Mechanical Engineering, Virginia Tech, \\ Blacksburg, VA \\ Gary D. Seidel \\ Aerospace and Ocean Engineering Department, Virginia Tech, Blacksburg, VA \\ Daniel J. Inman \\ Department of Aerospace Engineering, University of Michigan, Ann Arbor, MI
}

\begin{abstract}
For viscoelastic materials the stiffness and loss properties directly depend on not only strain but also strain rate and implicitly depend on temperature via time temperature superposition, which in case of harmonic loading leads to frequency dependent response. For viscoelastic composites in which at least one of the constituent materials is viscoelastic, there is great utility in the ability to predict the effective dynamic mechanical properties as a function of the constituent phase properties and geometry. The presence of different concentrations of nanofillers not only directly impacts the nanocomposite effective properties, but also contributes to the formation of an interphase layer, which also has a very strong influence on the effective properties. The interphase layer usually has properties which are distinct from that of the particle and the matrix phases. In this paper micromechanical methods combined with the correspondence principle of viscoelasticity are used to obtain the effective damping properties of viscoelastic composites. The interphase layer is modeled using the composite cylinder model and the composite sphere model and its effect on the overall stiffness and damping of the composite is investigated. Keywords: Nanocomposite; Interphase layer; Viscoelasticity; Micromechanics.
\end{abstract}

\section{Introduction}

One approach to calculate the effective viscoelastic properties of the composites is using the viscoelastic correspondence principle [Brinson, 2008] and the complex modulus [Hashin, 1970] of constituent materials to obtain the moduli of the composite. [Chandra, 2002, Hashin, 1970 , Hashin, 1970²] In this approach the correspondence principle of viscoelasticity helps to transform the time dependent properties of viscoelastic constituent materials into the frequency domain and finally to obtain the storage modulus and loss factor vs. frequency. Fiber reinforced and particle reinforced composites are two types of composite systems that are commonly investigated. The elastic-viscoelastic correspondence principle converts the time domain relaxation data of the viscoelastic material that is usually obtained by experiments to the frequency domain using the Carson transform [Schwarzl, 1967] which allows elasticity solutions to be applied in the Carson space. The interrelation between various time domain and frequency domain properties of viscoelastic materials is given in [Gross, 1953].

There are various micromechanical methods available to obtain the elastic properties of composites among which are the dilute, self-consistent and the Mori-Tanaka method [Mori, 1973] all of which are summarized in the micromechanics text by [Qu, 2006]. Traditionally, they all make use of the Eshelby equivalent inclusion method [Eshelby, 1957]. These methods when combined with the correspondence principle of viscoelasticity are capable of predicting damping in the composite. [Remillat, 2007] uses the self-consistent method to predict the damping of polymers filled with elastic particles. In the current study both the matrix and particle phases are assumed to be viscoelastic. The Mori-Tanaka method is used to find the effective properties and the correspondence principle of viscoelasticity is used to extend the micromechanics approach for viscoelastic constituents. Different combinations of elastic and viscoelastic materials are considered and the effective loss factor of the composites are obtained.

For nanoscale inhomogeneities an interphase layer is formed between the inhomogeneity and the matrix, which has 
different properties and behaves as a separate phase [Hernández-Pérez, 2010, Maligno, 2010, Jiang 2009]. The interphase layer also plays an important role in the dynamic properties of the nanocomposite [Paskaramoorthy, 2009, Qiao, 2009]. There are molecular dynamics methods available for predicting the behavior of this interphase layer [Liu, 2008]. Common nanoscale constituent material particles used in nanocomposites are: clay, silica, $\mathrm{TiO}_{2}$, $\mathrm{Au}$, ceramics, Magnesium, and CNT. Two major matrix materials are polymers and metals. Compared to metal matrix nanocomposites, polymer matrix nanocomposites possess a higher level of damping according to the viscoelastic behavior of the matrix. Depending on the particle and matrix molecular interactions the interphase layer may have different mechanical properties. In this study we obtained the interpase properties by interpolating between matrix and the reinforcing phase.

\section{Spherical inhomogeneities}

In this section a two phase composite consisted of spherical particles inside a matrix material is considered and the effective viscoelastic properties including storage modulus and loss factor is calculated as a function of frequency for different particle volume fractions. For more details on the micromechanics methods incorporated here, the reader is referred to [Qu, Cherkaoui, 2006].

The effective linear elastic stiffness tensor of the composite is

$$
\mathbf{L}^{\text {eff }}=\mathbf{L}^{N}+\sum_{r=1}^{N-1} c^{r}\left(\mathbf{L}^{r}-\mathbf{L}^{N}\right) \mathbf{A}^{r}
$$

In which $\mathbf{L}$ represents the stiffness tensors, superscript $r$ represents the $r$ th phase and $N$ is reserved for the matrix phase. $c^{r}$ is the volume fraction and $\mathbf{A}^{r}$ is the strain concentration tensor of the $r$ th phase.

The strain concentration tensor relates the average strain inside the $r$ th phase, $\overline{\boldsymbol{\varepsilon}}^{r}$ to the average strain of the whole composite $\overline{\boldsymbol{\varepsilon}}$ according to

$\overline{\boldsymbol{\varepsilon}}_{r}=\mathbf{A}^{r} \overline{\boldsymbol{\varepsilon}}$

In the present work the Mori-Tanaka method is used to estimate the strain concentration tensor for spherical inhomogeneities. According to this method we have

$$
\mathbf{A}^{r}=\mathbf{T}^{r}\left[c^{N} \mathbf{I}+\sum_{s=1}^{N-1} c^{s} \mathbf{T}^{s}\right]^{-1}
$$

where, $\mathbf{T}^{r}$ is the dilute strain concentration tensor of the $r$ th phase and relates the strain in the $r$ th phase, $\overline{\boldsymbol{\varepsilon}}^{r}$ to the applied strain $\boldsymbol{\varepsilon}_{A}$ on the representative volume element and is obtained from

$$
\mathbf{T}^{r}=\left[\mathbf{I}+\mathbf{S}^{r} \mathbf{M}^{N}\left(\mathbf{L}^{r}-\mathbf{L}^{N}\right)\right]^{-1}
$$

In which $\mathbf{I}$ is the identity tensor, $\mathbf{M}^{N}$, is the compliance tensor of the matrix, $\mathbf{L}$ is the stiffness tensors of the phases and $\mathbf{S}^{r}$ is the Eshelby tensor. The Eshelby tensor is defined for ellipsoidal inhomogeneities through the equivalent inclusion and relates the constrained strain of the inclusion, $\boldsymbol{\varepsilon}^{c}$ (when confined inside the matrix phase) to the free eigenstrain, $\boldsymbol{\varepsilon}^{T}$ (for example thermal strain, when the inclusion is unconstrained.

$$
\varepsilon^{c}=\mathbf{S} \varepsilon^{T}
$$

The Eshelby tensor depends on the geometry of the inhomogeneity particles and the material property of the matrix phase. [Mura, 1925]

In this section we investigate different composites made by different compositions of Epoxy and polycarbonate. The relaxation functions for these composites are obtained by performing a relaxation test using the DMA machine. The relaxation tests are done at several distinct temperature and the mastercurve is constructed according to the timetemperature superposition principle. The Relaxation mastercurves are shown in Fig (1)

Prony series are widely used to represent the relaxation modulus of viscoelastic materials as a function of time. The Prony series representation corresponds to the generalized Maxwell model for viscoelastic materials and is given by

$$
E(t)=E_{\infty}+\sum_{i=1}^{N} E_{i} \exp \left(-\frac{t}{\tau_{i}}\right)
$$




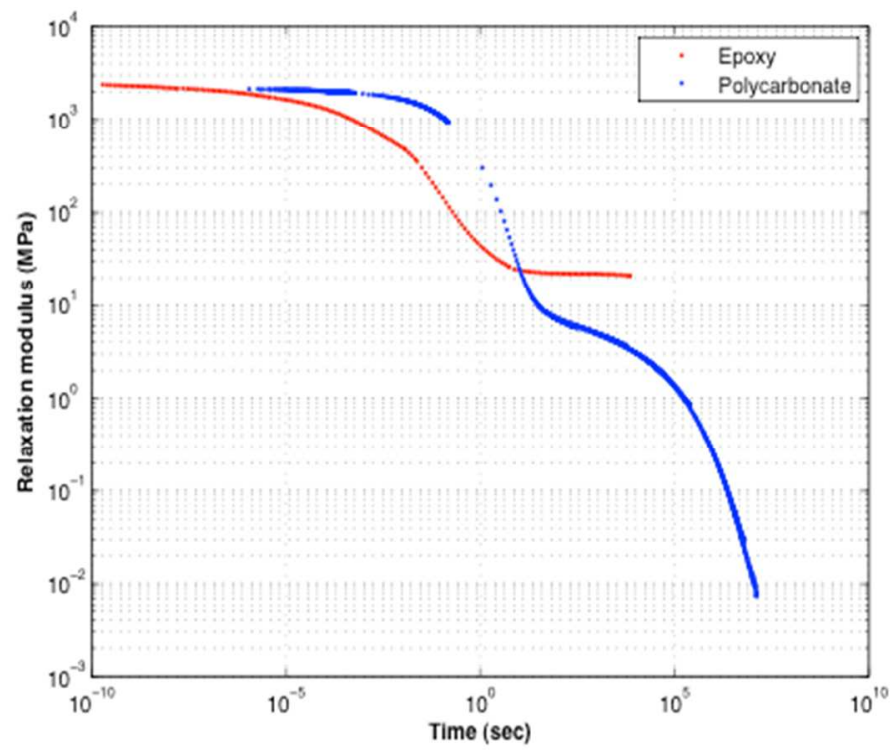

Figure 1. Relaxation function of epoxy and polycarbonate at $150 \mathrm{deg}$ C.

A Prony series is fit to the experimental data points using the nonlinear least square fitting algorithm [Bates, 1988] to obtain an analytical form of the relaxation function. For these relaxation functions 9-term Prony series are used to fit to the experimental data. The distribution of relaxation coefficients $\left(E_{i}\right.$ vs. $\left.\tau_{i}\right)$ of epoxy and PC are shown in Fig (2). Figure (3) shows the loss factor curves for different composites made from epoxy and polycarbonate.

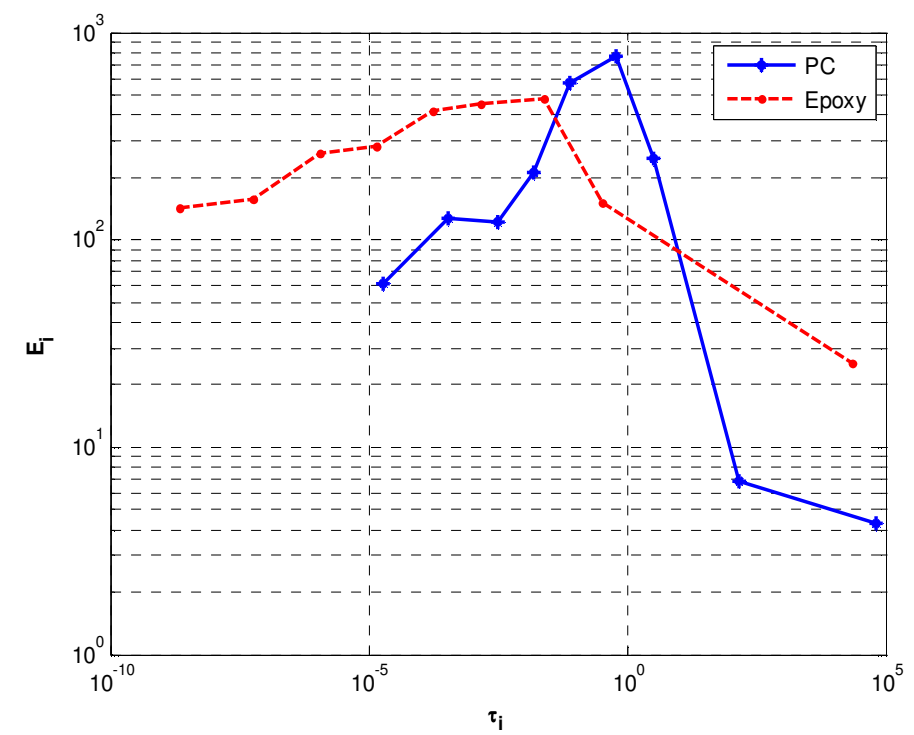

Figure 2. Relaxation spectra for epoxy and polycarbonate at $150 \mathrm{deg}$ C. 


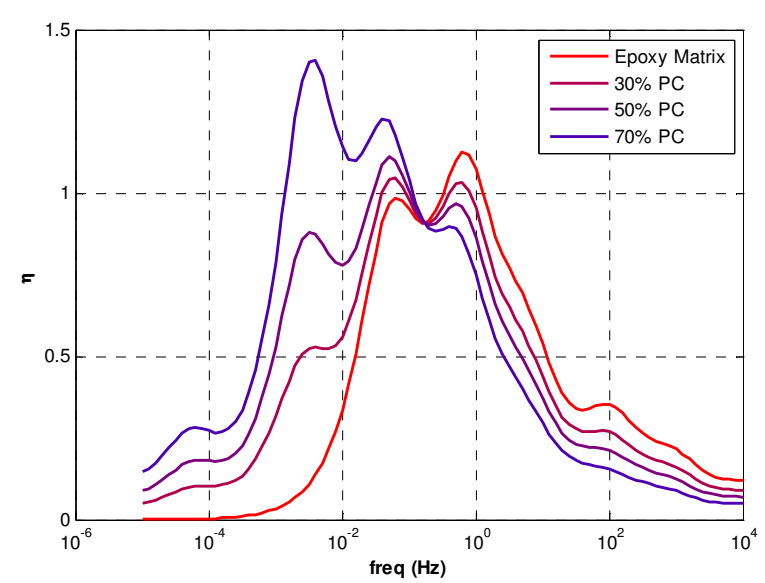

(a)

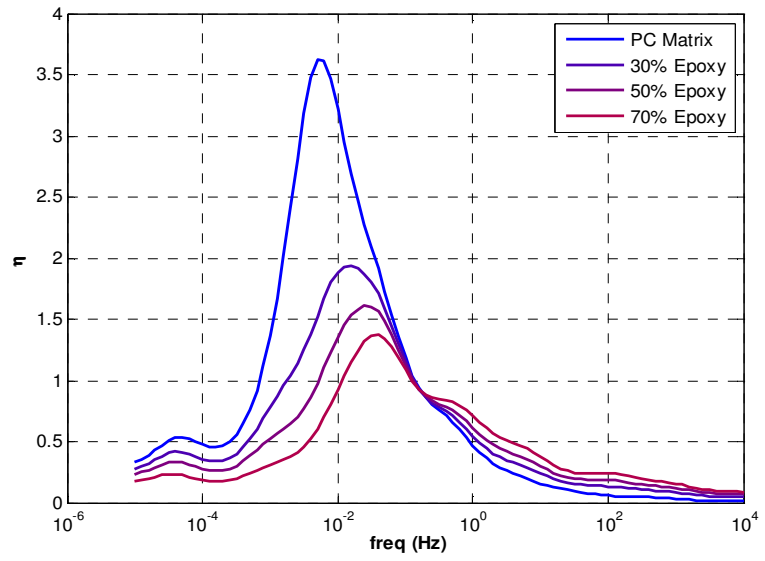

(b)

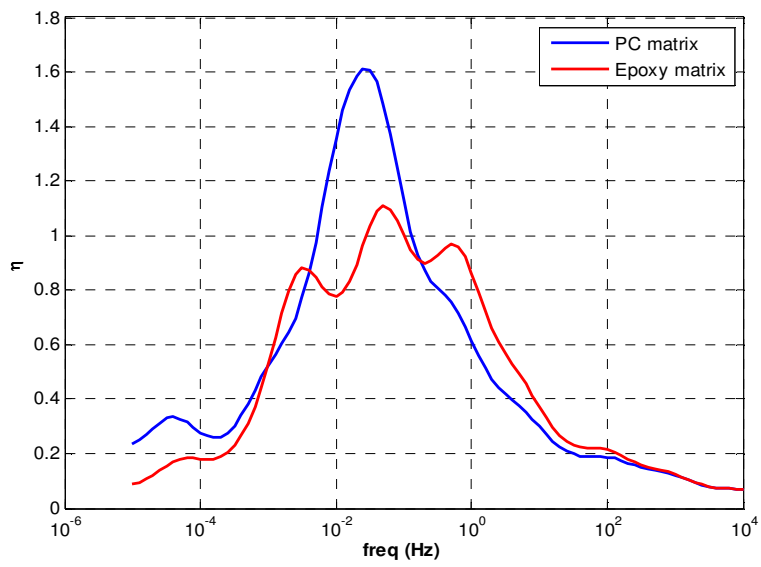

(c)

Figure 3. Loss factor of different composites made of polycarbonate and epoxy as a function of frequency for (a) different volume fractions of PC in epoxy as matrix (b) different volume fractions of epoxy in PC matrix (c) equal volume fractions of epoxy and PC

With increasing volume traction of particles in the matrix phase, the loss factor curves starts to reshape and gradually approach the pure particle curve. But the transition of the loss factor curve is not linear with respect to the particle volume fraction such that the composite loss factor curve remains closer to that of the matrix phase over the majority of the range of volume fractions. As shown in Fig (3c) although the constituent materials of the composite are the same, their effective loss factors have different patterns versus frequency. We explain this phenomenon as follows: Since the matrix is a continuous phase across the domain of the composite it contributes more to storage and dissipation of the strain energy. Therefore even if the volume fractions are the same, the matrix will dominate in the effective properties of the composite. This is why we see that for equal volume fractions of epoxy and polycarbonate there would be different loss factor patterns depending on the roles of the constituent materials.

\section{Spherical particles with interphase layer}

An interphase layer is always formed around any particle dispersed within a matrix by interaction between particle and matrix molecules. Depending on the molecular interactions between the two materials, the interphase layer may have different properties and thicknesses. This thickness is in the order of several nanometers and is independent of the particle dimensions. For large particles the interphase layer becomes negligible compared to the other phases present in the system, but for nanoparticles the interphase layer thickness is in the order of particle dimensions and becomes significant. 


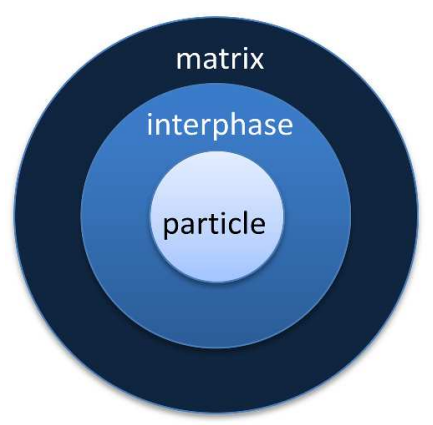

Figure 4. Composite sphere model with interphase layer

Here we consider a three phase material system shown in Fig (4) which is consisted ot a spherical particle covered by interphase layer within the matrix phase. The matrix phase is also modeled by an outer sphere layer with its radius dependent on the particle volume fraction according to:

$C_{p}=\left(\frac{r_{P}}{r_{M}}\right)^{3}$

The interphase layer itself, may be nonuniform. Depending on the particle surface and coating and molecular interactions its properties may change from point to point. Here we consider the case where the interphase layer is radially graded, starting from particle properties at $r=r_{P}$ and ending to matrix properties at $r=r_{M}$. In the other words we have

$M^{I}(r)=M^{P}+\left(M^{M}-M^{P}\right)\left(\frac{r-r^{P}}{r^{I}-r^{P}}\right)^{\beta}$

where $M$ could be replaced by any property and $\beta$ is the interphase exponent which determines how fast the properties are changed from particle to matrix within the interphase. Here we replace $M$ with relaxation function to obtain an estimate of the relaxation function of the interphase layer.

$E^{I}(r, t)=E^{P}(t)+\left(E^{M}(t)-E^{P}(t)\right)\left(\frac{r-r^{P}}{r^{I}-r^{P}}\right)^{\beta}$

For the matrix phase the relaxation function is available in the form of the Prony series. For the elastic particle (silica glass) the relaxation function is just the constant value of the elastic modulus of the material.

To simplify the calculation we make another assumption and replace the radially graded interphase layer with several layers with stepwise varying properties (Fig (5)).
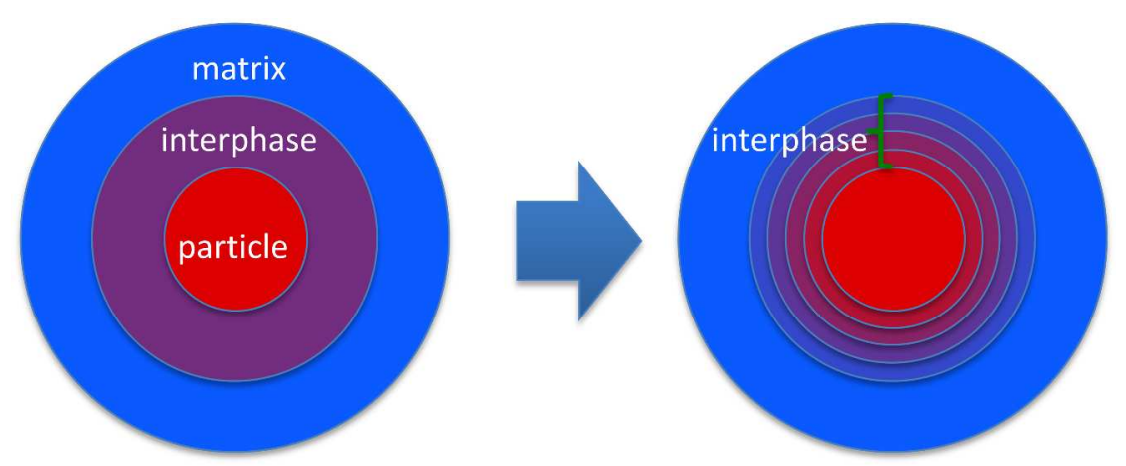

Figure 5. Descretizing the radially graded interphase 
Figure (6) shows the actual and interpolated relaxation functions and relaxation spectra for the phases in the system.

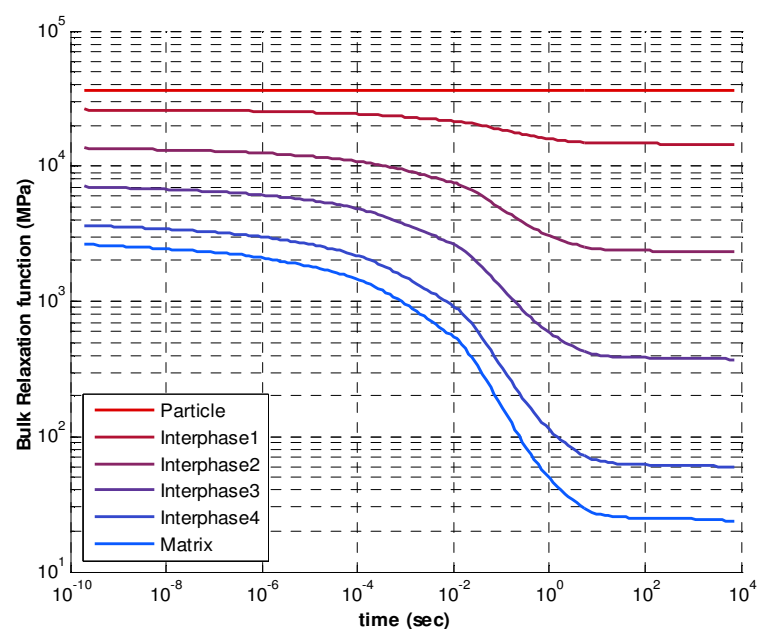

(a)

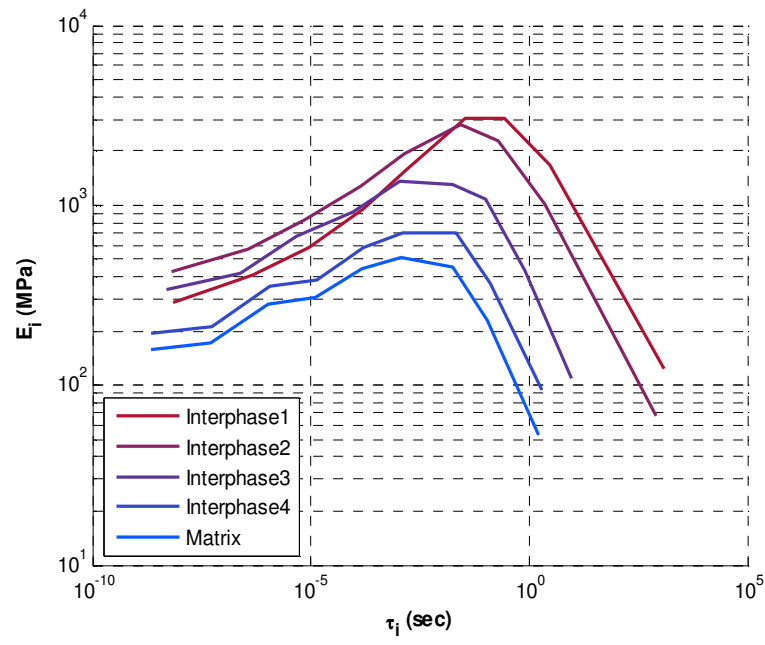

(b)

Figure 6. (a) Relaxation functions and (b) Relaxation spectra of the phases in the composite

According to the composite sphere model [Christensen, Lo] the effective bulk modulus of the multicoated inclusion is obtained from the following iterative equation

$$
\bar{K}_{n}=K_{n}+\frac{\left(r_{n-1}^{3} / r_{n}^{3}\right)\left(\bar{K}_{n-1}-K_{n}\right)\left(3 K_{n}+4 G_{n}\right)}{3 K_{n}+4 G_{n}+3\left(1-r_{n-1}^{3} / r_{n}^{3}\right)\left(\bar{K}_{n-1}-K_{n}\right)}
$$

in which $\bar{K}_{n}$ is the effective bulk modulus over the $n^{\text {th }}$ layer.

The bulk relaxation spectra of the constituent layers (particle, interphases, matrix) are in the form of

$$
K^{n}(t)=K_{\infty}^{n}+\sum_{i=1}^{N} K_{i}^{n} \exp \left(-\frac{t}{\tau_{i}^{n}}\right)
$$

where $\mathrm{n}$ changes over the layer numbers. According to the Carson transform, the complex modulus becomes

$$
K^{n^{*}}=K_{\infty}^{n}+\sum_{i=1}^{N} \frac{s K_{i}^{n}}{s+1 / \tau_{i}^{n}}
$$

Then, using the correspondence principle the complex effective bulk modulus of the composite is obtained from the following equation

$$
\bar{K}_{n}^{*}=K_{n}^{*}+\frac{\left(r_{n-1}{ }^{3} / r_{n}^{3}\right)\left(\bar{K}_{n-1}^{*}-K_{n}^{*}\right)\left(3 K_{n}^{*}+4 G_{n}^{*}\right)}{3 K_{n}^{*}+4 G_{n}^{*}+3\left(1-r_{n-1}{ }^{3} / r_{n}^{3}\right)\left(\bar{K}_{n-1}^{*}-K_{n}^{*}\right)}
$$

In case of harmonic loadings, by substituting the complex variable $s$ with $j \omega$ we find the bulk modulus as a function of frequency.

$$
K^{e f f *}(j \omega)=K^{\prime}(\omega)(1+\eta(\omega))
$$

Here we investigate the effective viscoelastic properties of a composite composed of silica glass particles within the epoxy matrix. Figure (7) shows the effective loss factor of the composite vs. frequency for different particle volume fractions and interphase exponents. 


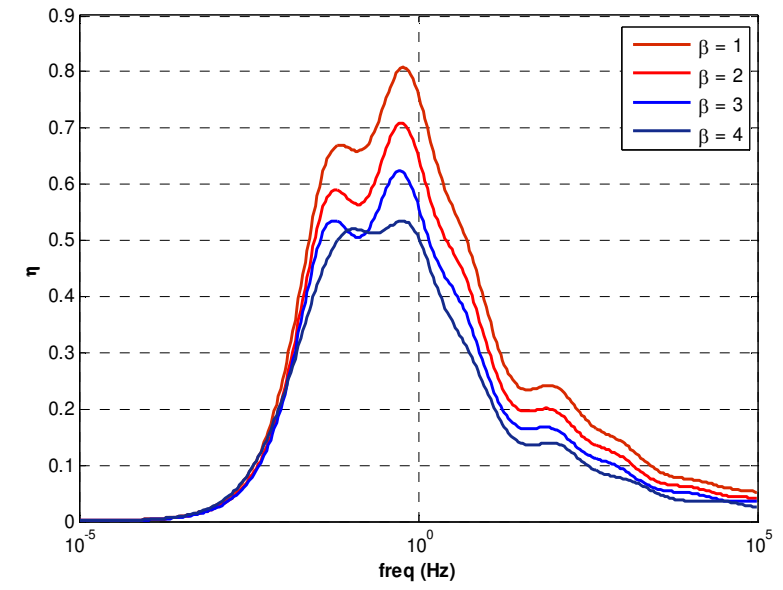

(a)

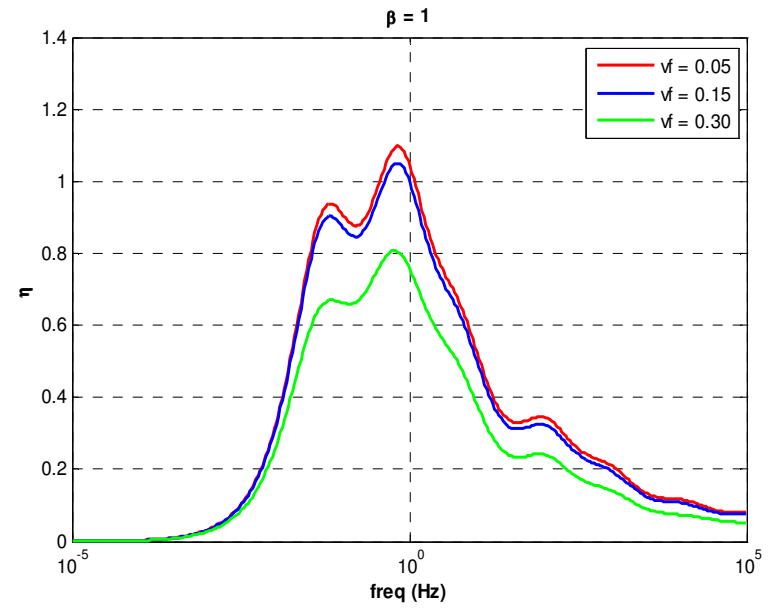

(b)

Figure 7. Effective loss factor of silica glass-epoxy composite as a function of frequency for (a) different interphase exponents, (b) different particle volume fractions

As shown in Fig (7) by increasing the volume fraction of the particles, the effective composite loss factor decreases. This is due to the fact that the interphase layer is less damped than the matrix and by increasing the particle volume fraction, more interphase would substitute for the matrix which results in a lower overall damping.

The interphase exponent $\beta$ determines how fast the properties in the interphase change from particle to matrix. Hence according to $\mathrm{Eq}(8)$, for bigger $\beta$ the interphase layer behaves more likely to the particle resulting in a lower loss factor.

\section{Cylindrical inhomogeneities with interphase layer}

Cylindrical reinforcements are of common inhomogeneities that are used to reinforce the composites. Either fibers or rod-shape particles fall into this category. Again like the spherical particles, these inhomogeneities may also contain a coating or be such small that form a significant interphase layer around them. Figure (8) shows an SEM image of the fuzzy fibers.

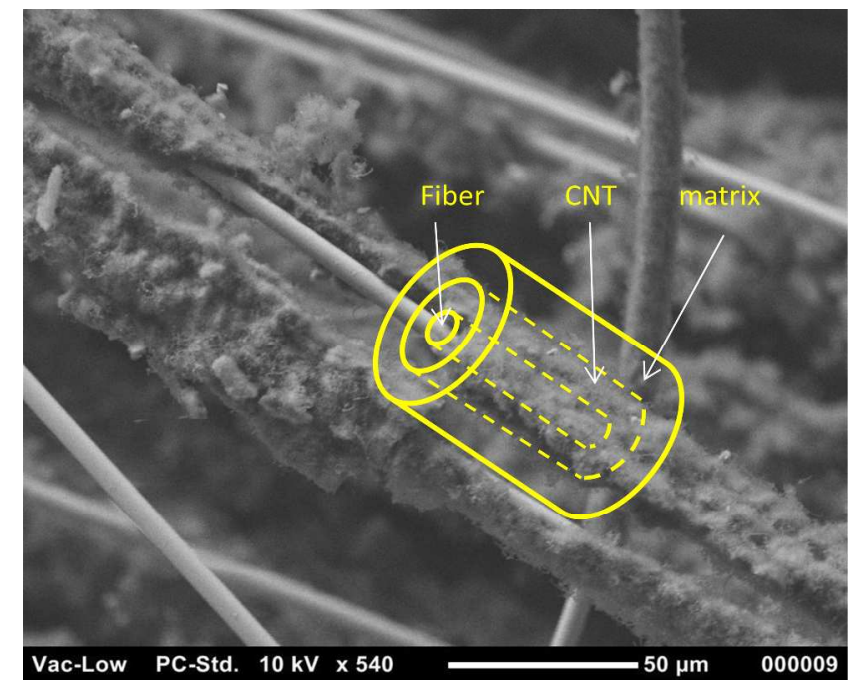

Figure 8. Composite cylinder model for fuzzy fiber composite 
These are glass fibers with a coating of carbon nanotubes which are used as reinforcing phase in some special composites. It is important to predict the effective viscoelastic properties of these composites in different deformations, i.e., tension or shear in different directions. This enables us to predict different modulus and damping values as a function of the frequency of loading.

The effective elastic properties are obtained by solving different boundary value problem on the composite cylinder model. Then, by applying the correspondence principle of viscoelasticity the elastic problem is converted to viscoelastic problem resulting in effective storage and loss moduli. Due to the cylindrical shape of the system, the material will be transversely isotropic resulting in 5 independent material properties. Of these properties we investigate longitudinal Young's modulus and in-plane bulk modulus here. Figure (9) shows the material model for three phase composite including fiber, interphase layer and the matrix.

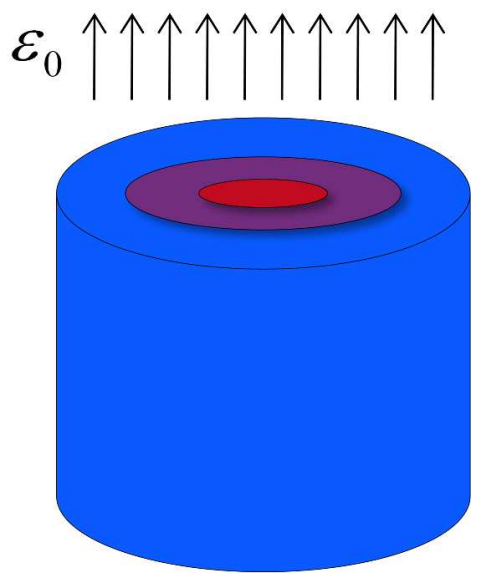

(a)

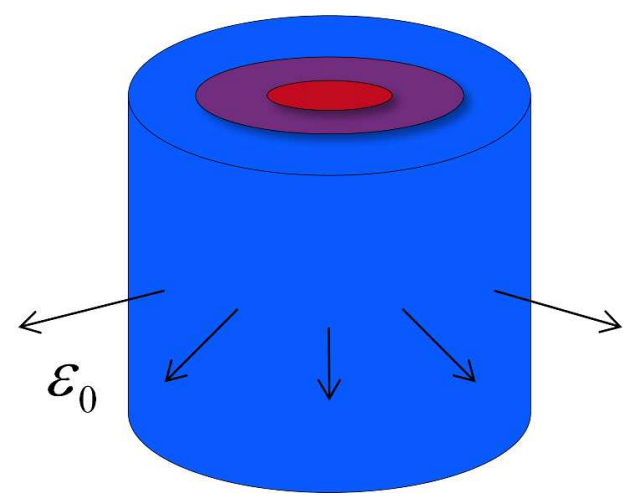

(b)

Figure 9. Material system for composite cylinder model (a) Longitudinal deformation (b) In-plane bulk deformation

\section{Longitudinal Young's modulus}

To find the longitudinal Young's modulus the composite cylinder is subjected to an axial load and the resulting axial deformation is calculated. The effective axial Young's modulus is then obtained by the ratio of the average axial stress to the average induced axial strain.

$E_{11}^{e f f}=\frac{\left\langle\sigma_{z z}\right\rangle}{\left\langle\varepsilon_{z z}\right\rangle}=\frac{\sum_{i=1}^{N}\left[\left(2 \mu^{i}+\lambda^{i}\right) \varepsilon_{0}+2 \lambda^{i} B_{1}^{i}\right]\left(r_{i}^{2}-r_{i-1}^{2}\right)}{\varepsilon_{0} r_{N}^{2}}$

Due to the applied strain, we first assume the following displacement field within the cylinder.

$u_{r}^{i}=B_{1}^{i} r+B_{2}^{i} 1 / r$

$u_{\theta}^{i}=0$

$u_{z}^{i}=\varepsilon_{0} z$

where $i$ corresponds to different layers and $B_{1}$ and $B_{2}$ are unknown parameters for each layer. The resulting nonzero strains are

$\varepsilon_{r r}^{i}=B_{1}^{i}-B_{2}^{i} 1 / r^{2}$

$\varepsilon_{\theta \theta}^{i}=B_{1}^{i}+B_{2}^{i} 1 / r^{2}$

$\varepsilon_{z z}^{i}=\varepsilon_{0}$ 
We assume that each phase is isotropic, therefore using the isotropic constitutive relations the following nonzero stresses are obtained.

$$
\begin{aligned}
& \sigma_{r r}^{i}=2 B_{1}^{i}\left(\mu^{i}+\lambda^{i}\right)-2 \mu^{i} B_{2}^{i} \frac{1}{r^{2}}+\lambda^{i} \varepsilon_{0} \\
& \sigma_{\theta \theta}^{i}=2 B_{1}^{i}\left(\mu^{i}+\lambda^{i}\right)+2 \mu^{i} B_{2}^{i} \frac{1}{r^{2}}+\lambda^{i} \varepsilon_{0} \\
& \sigma_{z z}^{i}=2 \lambda^{i} B_{1}^{i}+\left(2 \mu^{i}+\lambda^{i}\right) \varepsilon_{0}
\end{aligned}
$$

where $\lambda$ and $\mu$ are Lame' constants. Assuming perfect bonding between the layers, $\sigma_{r r}$ and $u_{r}$ will be continuous on the layer boundaries. It is also assumed that no traction is applied at the outer boundary and the stress and strain values are finite. Using these assumptions the unknown parameters in the displacement field are solved and the stress and strain fields within all the layers will be determined. Substituting the parameters into Eq (15) will finally give the longitudinal Young's modulus.

\section{In plane bulk modulus}

In this case a radial strain is applied to the cylinder and the radial stress is calculated. The in-plane bulk modulus is then calculated from

$$
K_{23}^{e f f}=\frac{\left(\mu_{N}+\lambda_{N}\right) r_{N}^{2} B_{1}^{N}-\mu_{N} B_{2}^{N}}{r_{N}^{2} B_{1}^{N}+B_{2}^{N}}
$$

The assumed displacement field is

$$
\begin{aligned}
u_{r}^{i} & =B_{1}^{i} r+B_{2}^{i} 1 / r \\
u_{\theta}^{i} & =0 \\
u_{z}^{i} & =0
\end{aligned}
$$

Then, the nonzero strain components are

$$
\begin{gathered}
\varepsilon_{r r}^{i}=B_{1}^{i}-B_{2}^{i} 1 / r^{2} \\
\varepsilon_{\theta \theta}^{i}=B_{1}^{i}+B_{2}^{i} 1 / r^{2}
\end{gathered}
$$

and the nonzero stresses are

$$
\begin{aligned}
\sigma_{r r}^{i} & =2 B_{1}^{i}\left(\mu^{i}+\lambda^{i}\right)-2 \mu^{i} B_{2}^{i} \frac{1}{r^{2}} \\
\sigma_{\theta \theta}^{i} & =2 B_{1}^{i}\left(\mu^{i}+\lambda^{i}\right)+2 \mu^{i} B_{2}^{i} \frac{1}{r^{2}} \\
\sigma_{z z}^{i} & =2 \lambda^{i} B_{1}^{i}
\end{aligned}
$$

Again, the same continuity and boundary conditions are applied and the unknown parameters are solved. Finally, Eq (19) gives the effective in-plane bulk modulus.

Equations (15) and (19) could be extended to viscoelastic case according to the correspondence principle of viscoelasticity. For this purpose instead of real valued quantities for Lame constants, complex values are used and finally, a complex effective modulus ( $K_{23}^{\text {eff* }}$ or $E_{11}^{\text {eff* }}$ ) is obtained for each of the loading cases. The complex moduli are then decomposed into storage and loss parts as previously shown in $\mathrm{Eq}(14)$.

A sample composite made of aligned glass fibers in an epoxy matrix is considered here. It is assumed that the fibers have a diameter of $7 \mu$ which are coated by an interphase layer of $5 \mu$ thick. The interphase layer for this case is assumed to be homogeneous. Interpolating the relaxation functions for fiber and matrix gives that of the interphase layer according to $\mathrm{Eq}(9)$. Figure (10) shows the relaxation functions and relaxation spectra for fiber, interphase and matrix. 


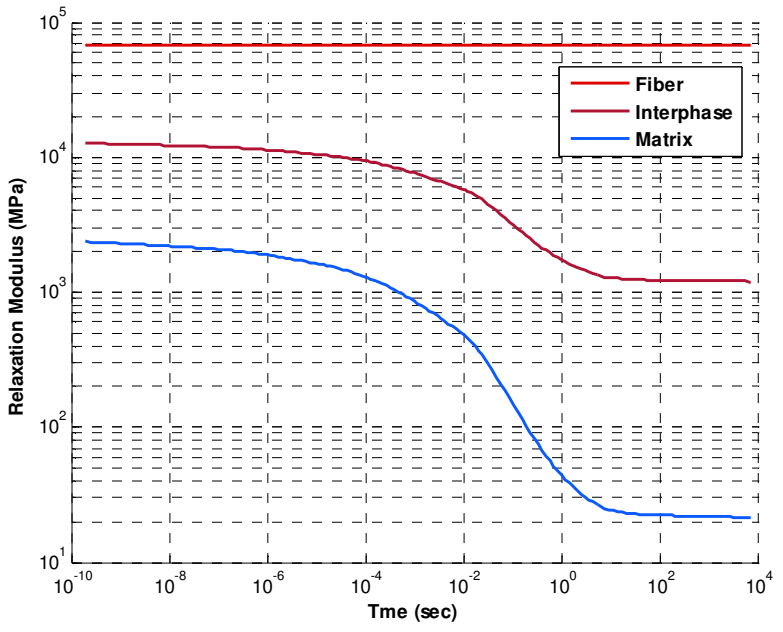

(a)

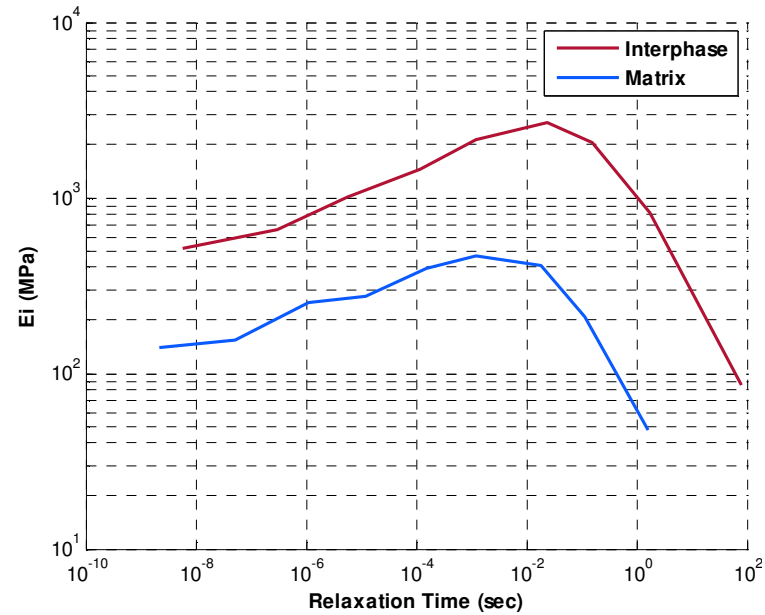

(b)

Figure 10. (a) Relaxation functions and (b) Relaxation spectra of the phases in the composite

Storage modulus and loss tactor tor both longitudinal elongation and lateral bulk detormation is plotted vs. frequency in Fig (11).

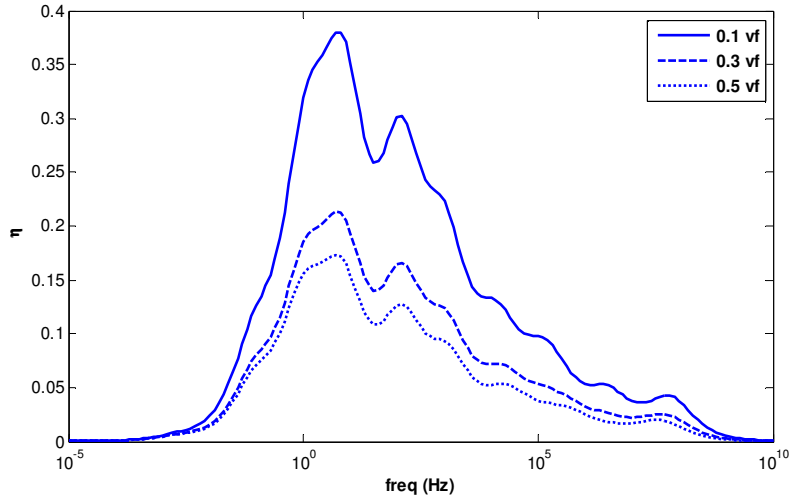

(a)

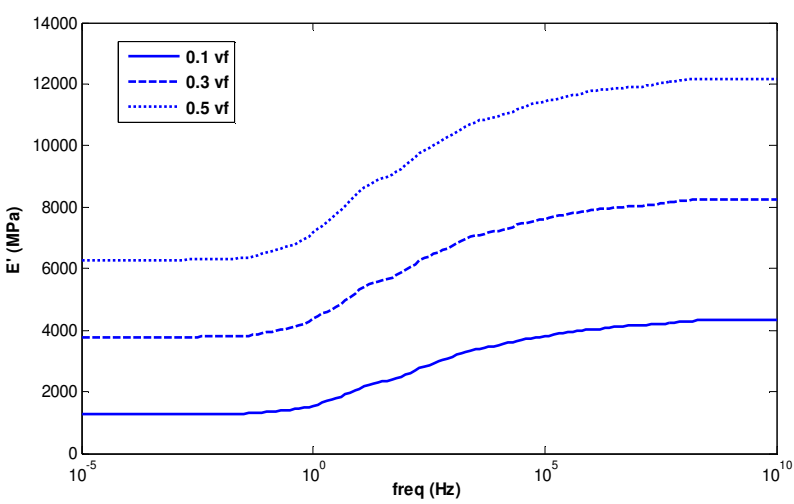

(c)

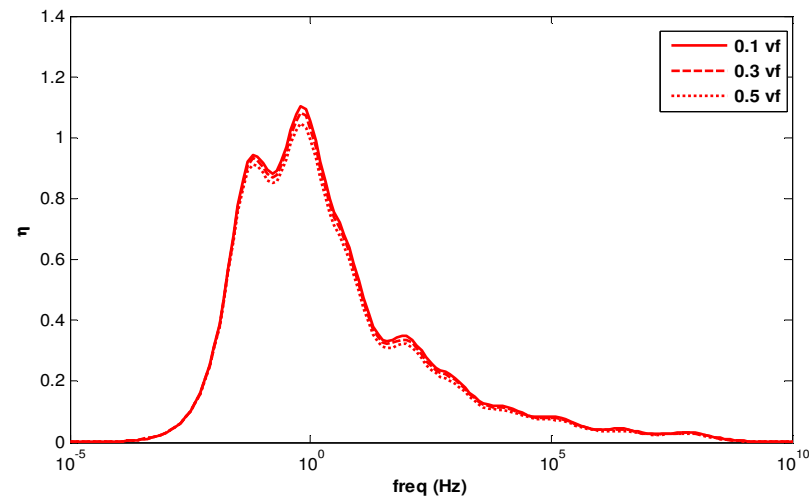

(b)

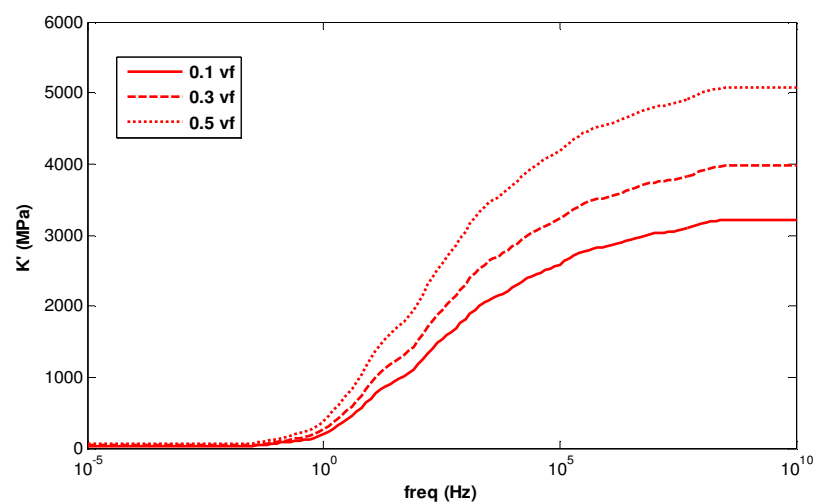

(d)

Figure 11. (a, c) Longitudinal elongation (b, d) In-plane bulk deformation storage modulus and loss factor of composite containing aligned fibers with interphase 
In longitudinal deformation both the storage modulus and the loss factor are affected significantly by the fiber volume fraction. That is because the fibers are aligned in the deformation direction and contribute directly to the load transfer. Hence, their effect is more like the "rule of mixtures" behavior. On the other hand we see that for the in-plane bulk deformation the loss factor does not change at all. That is because in this case most of the deformation occurs in the matrix phase

\section{Conclusions}

In this study we combined micromechanics with the correspondence principle of viscoelasticity to obtain the effective viscoelastic properties of composites containing spherical or aligned fibers. Our procedure requires the relaxation functions of the constituent materials and gives the storage modulus and the loss factor of the composite as a function of the frequency of loading. We also investigated the presence of the interphase layer on different storage modulus and loss factor curves. The results are shown for different volume fractions of the reinforcement phase. We showed that with an interphase layer with properties between the matrix and the inhomogeneity, the effective loss factor of the composite will decrease by increasing the particle volume fraction.

\section{ACKNOWLEDGEMENTS}

The authors greatly acknowledge the support of AFOSR through MURI-18 Synthesis Characterization and Modeling of Functionally Graded Hybrid Composites for Extreme Environments (Contract/Grant \#: FA-9550-090686).

\section{References}

[1] Adolf D.B., Chambers R.S., Caruthers J.M., Extensive validation of a thermodynamically consistent, nonlinear viscoelastic model for glassy polymers, Polymer, 45 (2004) 4599-4621.

[2] Bates D.M., Watts D.G., Nonlinear regression analysis and its applications, New York : Wiley, 1988.

[3] Brinson H.F., Brinson L.C., Polymer Engineering Science and Viscoelasticity, An Introduction, Springer, 2008.

[4] Chandra R., Singh S.P., Gupta K., Micromechanical damping models for fiber-reinforced composites: a comparative study, Composites: Part A, 33 (2002) 787-796.

[5] Eshelby J.D., The determination of the elastic field of an ellipsoidal inclusion, and related problems, Proceedings of the Royal Society of London. Series A, Mathematical and Physical Sciences, Vol. 241, No. 1226 (Aug. 20, 1957), pp. 376-396

[6] Ferry J.D., Viscoelastic Properties of Polymers, New York, Wiley, 1980

[7] Gross B., Mathematical structure of the theories of viscoelasticity, 1953.

[8] Hashin ZVI, complex moduli of viscoelastic composites-I. General theory and application to particulate composites, Journal of Solids and Structures, $6\left(1970^{1}\right)$ 539-552.

[9] Hashin ZVI, complex moduli of viscoelastic composites-II. Fiber reinforced materials, Journal of Solids and Structures, $6\left(1970^{2}\right) 797-807$.

[10] Mori T., Tanaka K., Average stress in matrix and average elastic energy of materials with misfitting inclusions, Acta Metall, 21 (1973) 571-574.

[11] Mura T., Micromechanics of Defects, 1925.

[12] Qu J., Cherkaoui M., Fundamentals of Micromechanics of Defects, Wiley, 2006.

[13] Remillat C., Damping mechanism of polymers filled with elastic particles, Mechanics of Materials 39 (2007) 525-537.

[14] Schwarzl F.R., Struik L.C.E., Analysis of relaxation measurements, Advances in Molecular Relaxation Processes, 1 (1967-68) 201-255.

[15] Seidel G.D., Lagoudas D.C., A micromechanics model for the electrical conductivity of nanotube-polymer nanocomposites, Journal of Composite Materials, Vol. 43, No.9 (2009) 917-941.

[16] Sperling L.H., Introduction to Physical Polymer Science, $3^{\text {rd }}$ edition, Wiley, 2001.

[17] A. Hernández-Pérez, F. Avilés, Modeling the influence of interphase on the elastic properties of carbon nanotube composites, Computational Materials Science, Vol 47, Issue 4, (2010), 926-933.

[18] A.R. Maligno, N.A. Warrior, A.C. Long, Effects of interphase material properties in unidirectional fibre reinforced composites, Composites Science and Technology, Vol 70, Issue 1, January (2010), Pages 36-44. 
[19] Yunpeng Jiang, Keiichiro Tohgo, Yoshinobu Shimamura, A micro-mechanics model for composites reinforced by regularly distributed particles with an inhomogeneous interphase, Computational Materials Science, Vol 46, Issue 2, August 2009, 507-515.

[20] R. Paskaramoorthy, S. Bugarin, R. Reid, Effect of an interphase layer on the dynamic stress concentration in a Mg-matrix surrounding a SiC-particle, Composite Structures, Vol 91, Issue 4 (2009), 451-460.

[21] Rui Qiao, L. Catherine Brinson, Simulation of interphase percolation and gradients in polymer nanocomposites, Composites Science and Technology, Vol 69, Issues 3-4, March (2009), 491-499.

[22] Y.J. Liu, N. Nishimura, D. Qian, N. Adachi, Y. Otani, V. Mokashi, A boundary element method for the analysis of CNT/polymer composites with a cohesive interface model based on molecular dynamics, Engineering Analysis with Boundary Elements, Volume 32, Issue 4, April (2008), Pages 299-308 